\title{
Detection of Organic Pollutants with a Pulsed Ion Mobility Spectrometer
}

\author{
Frank Gunzer
}

\begin{abstract}
Ion Mobility Spectrometry (IMS) is a well known technique for the detection of trace gases in ambient air. Portability due to its small size, high sensitivity in the ppbv range combined with fast response times of a few seconds make IMS devices the preferred detectors for hazardous substances. These range from drugs and explosives over pollutants in e.g. industrial plants to chemical warfare agents. The analytes are ionized by positve and negative reactant ions which have been produced in ambient air with help of free electrons. Certain analytes are then present as positive ions, others as negative ions, depending on their chemical properties. While pollutants forming positive ions in the device have been intensitvely investigated in the last $\mathbf{2 0}$ years, the investigation for pollutant ions detected in the negative mode has concentrated mainly on the detection of explosives. This paper describes the characteristics of analytes in the negative mode of an IMS device when detected with a novel pulsed ion mobility spectrometer.
\end{abstract}

Index Terms-Cluster formation in ambient air, ion mobility spectometry, pulsed electron beams

\section{INTRODUCTION}

Ion Mobility Spectrometry is a standard technique used to analyze ambient air [1]. The advantages are a small detector size (typically consisiting of a tube which is ca. $8 \mathrm{~cm}$ long with a diameter of ca. $2 \mathrm{~cm}$ ), a high sensitivity in the ppb range and a fast response time of a few seconds. The information an IMS device provides is the mobility of the analytes, which depends on many parameters such molecule size and weight. This information is obtained by ionizing analyte molecules and accelerating them with help of electric fields. Since the device is operated under ambient conditions, the accelerated ions collide with the air molecules and reach a mobility dependent drift velocity. The ions fly through a drift region of a certain length and the produce an electric signal at the end. By measuring the time the ions spent in the drift region their mobility can thus be calculated.

The ionization of the analyte molecules is achieved with help of reactant ions. These are formed by using free electrons that react with air molecules, especially nitrogene. The results are $\mathrm{H}_{3} \mathrm{O}^{+}$clusters and $\mathrm{O}_{2}^{-}$clusters. These react with the analyte molecules, and depending on their chemical and physical properties, either positvely charged analyte ions are formed (by e.g. proton transfer) or negatively charged ones (by e.g. proton abstraction). The device detects only one type of ions, which can be selected via the polarity of the

Manuscript received December 31, 2012; revised February 18, 2013.

Frank Gunzer is with the Information Engineering and Technology Faculty, German University in Cairo, Cairo, Egypt 11853 (e-mail: frank.gunzer@guc.edu.eg). pulse that aceelerates the ions into the drift region, and thus the device is either operating in the so called negative mode when it detects the negatively charged ions, or in the positive when it detects the positively charged ions [2].

The free electrons are provided by a source, which is in standard devices a $\beta$-radiating radioactive source. Since it produces free electrons continuously and thus the ionization is a continuous process, devices operating with such sources are called to work in a continuous mode. Our device uses a novel electron gun that can be operated in pulsed mode, i.e. the electrons are emitted in short pulses with a certain duration and intensity. Thus not only the electron beam can be controlled (especially regarding electron energy and beam intensity), but furthermore delay times can be introduced in-between ionization and detection, in which the analytes can recombine with an analyte depending rate.

From standard IMS devices operating in positive mode it is known that not only monomer signals of the analytews form, but also dimer signals. The monomer signals are thed protonated analyte ions, while the dimer signals are proton stabilized dimers of analyte molecules. Both can form furthermore water cluster with water molecules [2]:

$$
\begin{aligned}
& M+H^{+}\left(H_{2} \mathrm{O}\right)_{n} \rightarrow \mathrm{MH}^{+}\left(\mathrm{H}_{2} \mathrm{O}\right)_{n-x}+x \mathrm{H}_{2} \mathrm{O} \\
& M H^{+}\left(\mathrm{H}_{2} \mathrm{O}\right)_{n}+\mathrm{M} \rightarrow \mathrm{M}_{2} \mathrm{H}^{+}\left(\mathrm{H}_{2} \mathrm{O}\right)_{n-x}+x \mathrm{H}_{2} \mathrm{O} \\
& M H^{+}\left(\mathrm{H}_{2} \mathrm{O}\right)_{n}+\mathrm{N} \rightarrow \mathrm{MNH}^{+}\left(\mathrm{H}_{2} \mathrm{O}\right)_{n-x}+x \mathrm{H}_{2} \mathrm{O}
\end{aligned}
$$

The dimer ions have a different mobility and thus form an extra signal. Also the appearance of asymmetric dimers is known (eq. 3). If dimer peaks appear or not is a matter of analyte concentrationn and only at low concentrations (depending on the substance, for DMMP e.g. it is $30 \mathrm{ppmv}$ ) only the corresponding monomer peak appears. The IMS spectrum of two substances thus can show is the worst case six peaks: One for the reactant ions, one for each monomer, one for each dimer, and one for the asymmetric dimer [3]. For three substances it would be accordingly ten peaks. Fig. 1 shows a spectrum for a mixture of dimethylformamid, ethyl methyl ketone and diisobutyl ketone. Since the selectivity of IMS devices is normally low, these peaks can not automatically identify the substances. In order to simplify the interpretation, at least knowing which peaks are formed by dimers and which by monomers could simplify the task. We could show that using delay times and investigating the decay behavior can seperate these diferent peaks, i.e. it is clear which peaks are monomer signals and which are (asymmetric) dimer peaks. In Fig. 1 this shown with the dashed line where due to a delay of two milliseconds only the dimer peaks are left in the spectrum. It showed that the proton affinity has an 
influence, too, since the dimer peak of the analyte with the highest proton affinity decays most slowly. Especially in environmental monitoring and pollution monitoring, where it is common to have many analytes present in a device, this helps to improve the performance of IMS detectors.

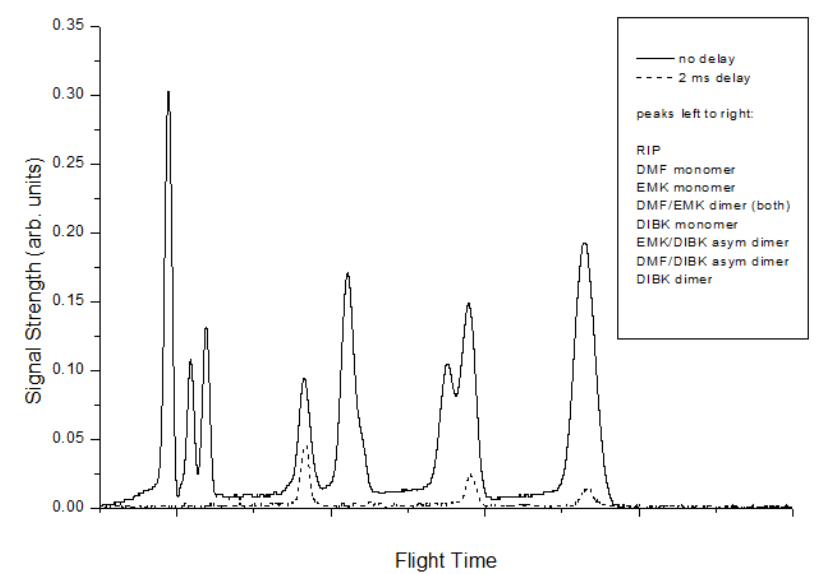

Fig. 1. Example for the complexity of an IMS spectrum with three substances recorded in the positive mode for dimethylformamid (DMF), ethyl methyl ketone (EMK) and diisobutyl ketone (DIBK). For DMF and EMK the dimers have almost the same flight time but are still distinguishable, while their asymetric dimer is not visible.

In the negative mode, it is proton abstraction caused by $\mathrm{OH}^{-}$ions (formed by $\mathrm{O}_{2}^{-}$clusters) that leads to an ionization of the analyte [2]:

$$
\mathrm{M}+\mathrm{OH}^{-}\left(\mathrm{H}_{2} \mathrm{O}\right)_{n} \rightarrow\left(\mathrm{M}-\mathrm{H}^{-}\right)\left(\mathrm{H}_{2} \mathrm{O}\right)_{n+1-x}+x \mathrm{H}_{2} \mathrm{O}
$$

Proton bound dimer formation as in the case of the positive mode is here not possible. The standard literature does not describe the dimer formation in the negative mode. The simplest possibility that can lead to negatively charged ions including more than one analyte moelcule would be a cluster of an analyte anion $\left(M-H^{-}\right)$with a neutral (similarly for asymmetric clusters; water clusters not included):

$$
\begin{gathered}
\left(M-H^{-}\right)+M \rightarrow M\left(M-H^{-}\right) \\
\left(M-H^{-}\right)+N \rightarrow N\left(M-H^{-}\right)
\end{gathered}
$$

The negative mode could thus suffer from the same problems regarding more than one peak per analyte as the positive mode. The investigation of analytes in the negative mode has been done only with limited effort in the last 20 years, while for the positive mode a lot of publications exist. One reason might be that a lot of substances that form preferably negatively charged analyte ions contain halogene atoms, which easily fragment so that the spectra always contain signals from these atoms, which depending on their concentration can also trigger side reactions with the ions present. Here we present the preliminary results of investigations with the substances formic acid and 2-Chlorophenol (single substances and mixture of different concentrations) which are important substances to be monitored in chemical plants. After a short description of the set up we will show spectra of these substances obtained with a pulsed IMS device in negative mode and discuss the characteristics of these spectra. With help of quantum chemical calculations we will then further investigate the presence and absence of certain analyte peaks in the spectrum.

\section{EXPERIMENTAL}

The set up has been described in previous publications [4-6] so that here only a general overview will be given. A typical IMS device consists of a tube of ca. $8 \mathrm{~cm}$ length with three distinct sections, each having its own electric field. The first section, normally $3 \mathrm{~mm}$ wide, is called reaction region, in which the free electrons indirectly ionize the analyte. A weak electric field of a few $\mathrm{V} / \mathrm{cm}$ prevents the ions from drifting into the next section, which is called the drift regions. The ions are accelerated by an electric pulse from the reaction region into the ca. $5 \mathrm{~cm}$ long drift region, where they experience an electric field of a few $100 \mathrm{~V} / \mathrm{cm}$ that accelerates the ions towards the third region, the detector region. This field leads to a substance specific drift velocity due to collisions with ambient air molecules, so that different analytes reach the end of this section after different drift times. In the detector region, normally $2 \mathrm{~mm}$ wide, a stronger electric field accelerates the ions towards the detector. For pulsed operation, the free electrons in the reaction region are created by an electron gun, which allows full control over the electron beam in terms of intensity and duration and allows thus the introduction of delay times. The instrumental difference in comparison with standard IMS devices which use typically radioactive substances to continuously produce free electrons are thus rather small. The response is therefore similar to standard IMS, the possibility to use the decay characteristic as additional orthogonal parameters being the decisive difference.

Different analyte concentrations are generated by mixing dry air (water concentration at $292 \mathrm{~K}$ below 200 ppbv, dew point of $181.1 \mathrm{~K}$ ) with a gas mixture composed of dry air and analytes (generated via gas evaporation in a permeation oven into a constant air flow of $100 \mathrm{ml} / \mathrm{min}$ ). The concentrations have been verified with an Ion Science Tiger Select PID detector and a Draeger CMS. For single substances a total mixed flow of $230 \mathrm{ml} / \mathrm{min}$ is used for each substance. The sample gas flow into the reaction region of the IMS is 40 $\mathrm{ml} / \mathrm{min}$. For the investigation of a formic acid/ 2-Chlorophenol mixture $115 \mathrm{ml} / \mathrm{min}$ of the formic acid sample gas flow and $115 \mathrm{ml} / \mathrm{min}$ of the 2-Chlorophenol sample gas flow are mixed together giving the final gas flow of $230 \mathrm{ml} / \mathrm{min}$.

\section{RESUlTS AND DiSCUSSION}

The first spectrum to be discussed is that of 2-chlorophenol. This analyte is an example for a negative mode IMS spectrum showing, similar to the positive mode, two peaks for the analyte. The upper spectrum shows the development of the analyte peaks when increasing the concentration. The leftmost peak is the reactant ion peak (RIP), followed by the analyte monomer peak and a peak that behaves similar to dimer peaks in the positive mode (where the dimer peaks show only at higher concetrations, increasing their intensity 
with increasing concentration while the monomer peaks increase until the dimer peaks appear, and then lose intensity while the dimer peaks increase in intensity). The lower spectrum shows the decay behavior when introducing a delay time between ion production and detection. In the positve mode the RIP decays fastest, the monomer peaks decay more slowly than the RIP but faster than dimer peaks [7]. So again the peak at ca. $10.3 \mathrm{~ms}$ flight time shows a behavior as it is known from dimers in the positve mode. Thus our conclusion is that a cluster formation is leading to this peak. The simplest way possible would be a cluster formed by a 2-chlorophenol anion and a neutral 2-chlorophenol molecule. Thus we performed quantum chemical calculations in order to verify if such clusters could form. The difficulty is that these calculations assume vacuum conditions and neglect the influence of water clusters. A similar problem exists when using the proton affinity to explain certain observations in ion mobility spectrometers, but there at least a similar tendency has been observed, so we assume a similar tendency with repect to the results of the calculations, but the results have to be interpreted with care.

We have performed calculations with geometry optimization and thermodynamic corrections calculated at the B3LYP/6-31(d) level of theory using a scaling factor of 0.9804 and calculating the single point energy at the B98/6-311++(3df,3dp) level of theory. The B98 functional achieved at least similar results to the well known B3LYP functional but achieving higher precision for larger molecules when applied to the G3/05 molecule set [8] (average deviation from experimental values for formation enthalpies when using B3LYP/6-31(d) optimized geometries less than $3 \mathrm{kcal} / \mathrm{mol}$, for hydrogen-bonded cluster even below $1 \mathrm{kcal} / \mathrm{mol})$. The result was that such a cluster would have a stabilization of $-15 \mathrm{kcal} / \mathrm{mol}$, which is in the order of magnitude of proton bound dimers in the positive mode. Thus we interpret the signal at $10.3 \mathrm{~ms}$ flight time to be the result of such a cluster. Fig. 2 shows that the advantages of different decay times between monomer and dimer signals established for the positive mode can also be used in the negative mode for the discrimination of monomer and cluster signals belonging to the same analyte.

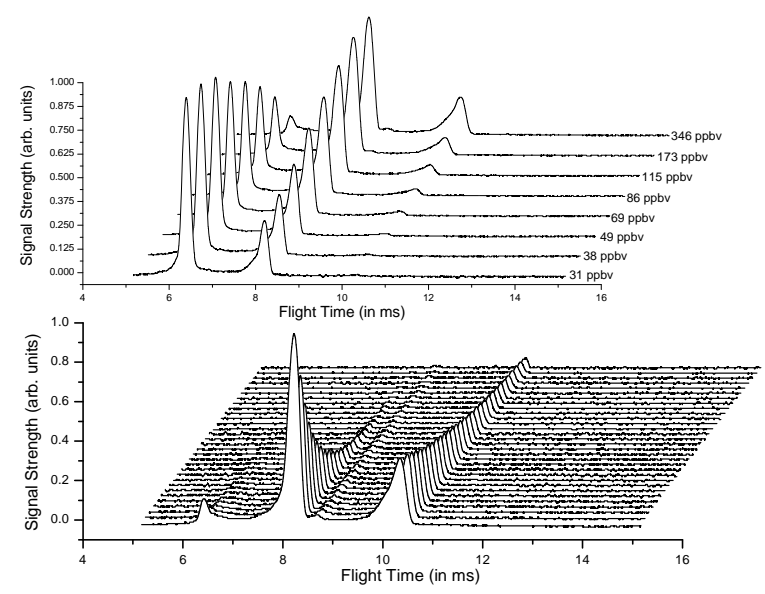

Fig. 2. IMS spectrum of 2-chlorophenol showing the development of the peaks when increasing the intensity (upper graph), and when increasing the delay time between ionization and detection (lower graph, concentration 346 ppmv, delay time ranging from $0 \mathrm{~ms}$ to $3 \mathrm{~ms}$ ).
A second substance that has been investigated is formic acid. This chemical is used in many industrial processes and although only slightly toxic, it is nevertheless for example listed in the Toxics Release Inventory List introduced in the United States as part of the Pollution Prevention Act from 1986. In IMS spectra formic acid gives strong signals. Fig. 3 shows the corresponding spectrum in the upper graph. Even for high concentrations, there is only one peak at the same position, which is the monomer peak of formic acid. At high concentrations the reactant ions are completely gone so there is no RIP peak, at low concentrations there are still reactant ions left after the ionization of the analyte molecules and thus there is a RIP peak (which has in general a faster flight time than any other analyte). The graph below shows the decay curve, as usual the RIP decays faster than the analyte peak. In the positive mode it is possible to make dimer peaks visible when using longer delay times even when they nomally do not appear because the concentration is too low. But here even under these conditions no other peak appears.

Quantum chemical calculations again at the B98/6-311++(3df,3dp)// B3LYP/6-31(d) level of theory yield a stabilization for a formic acid anion clustering with a formic acid neutral molecule of $36 \mathrm{kcal} / \mathrm{mol}$. This value is in good agreement with a value of $27 \mathrm{kcal} / \mathrm{mol}$ calculated at the B3LYP/6-31G+(d,p)//B3LYP/6-31+G(d,p) level of theory [9] which has a mean absolute deviation from experimental values of $3.9 \mathrm{kcal} / \mathrm{mol}$ with a standard deviation of 3.2 $\mathrm{kcal} / \mathrm{mol}$ when applied on the G2 molecule set (B3LYP: 2.7 $+/-2.6 \mathrm{kcal} / \mathrm{mol}$ ) [10]. An intesresting fact is that the optimized structure obtained was one where the neutral formic acid molecule loses its hydrogen atom so that finally a proton bound cluster of two formate anions is obtained. This process would be similar to the proton bound dimer formation binding two neutral analyte molecules in the positive mode, only that here in the negative mode it would be two anions bound by a proton. For the other molecules investigated here we could not obtain such a cluster.

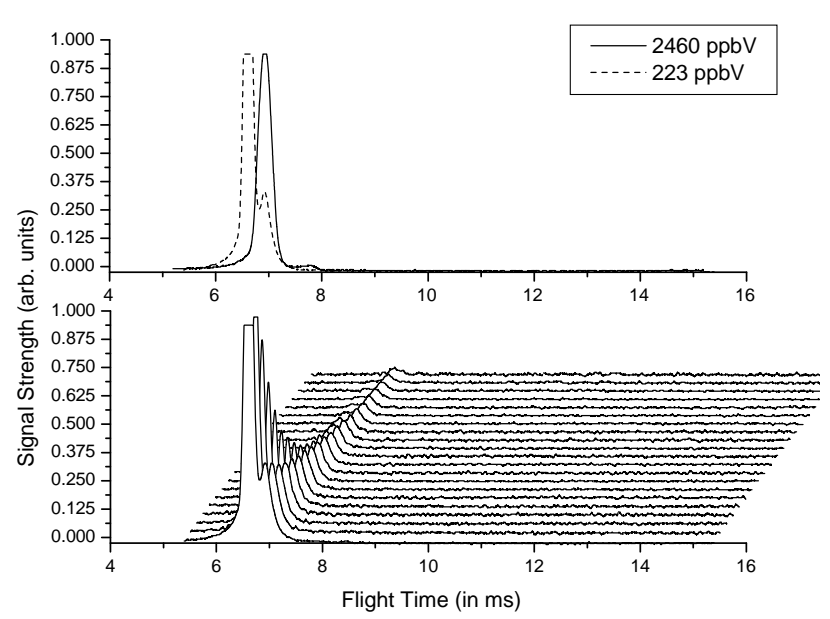

Fig. 3. IMS spectrum of formic acid (upper graph) which shows only a monomer peak at $6.9 \mathrm{~ms}$ flight time (and the RIP at low concentrations left of it). Also at longer delay times no other peaks appear (lower graph, delay ranges from 0 to $2 \mathrm{~ms}$ ).

Finally, we have investigated a mixture of both substances in order to find out to w hich extent the behavior would be 
similar to that of mixtures in the positive mode. One important difference is the absence of a second dimer signal, or here of an anion/neutral cluster. Fig. 4 shows the corresponding specra for two concentrations of 2-chlorophenol (upper graph: 85 ppbv, lower graph: 275 ppbv). As can be seen, the single substance peaks are present, the 2-chlorophenol cluster peak appears only at high analyte concentration (peak 5). There is only one extra peak (peak 4) with a longer decay time than the monomer peaks. Thus we conclude that this peak is again a cluster of two analyte moelcules, but this time formed by both analytes, similar to the asymmetric dimer formation in the positive mode. Calculations at the B98/6-311++(3df,3dp)// B3LYP/6-31(d) level of theory yield a interesting optimized structure: Independent from whether the formate anion clusters with a 2-chlorophenol neutral or vice versa, the optimized structure is that both analytes are present as anions bound by a proton in-between. This structure reminds again of the proton bound dimer formation in the positive mode, but might here be a special case due to the acidic properties of formic acid. The stabilization energies are $30 \mathrm{kcal} / \mathrm{mol}$ when 2-chloropehnol is present as an anion, and $34 \mathrm{kcal} / \mathrm{mol}$ when the formate anion is present in the cluster. The difference is too small regarding the error margins to help deciding which process actualy leads to the optimized structure. Finally, this spectrum shows that such cluster peaks show a longer decay time than monomer peaks also in the negative mode.

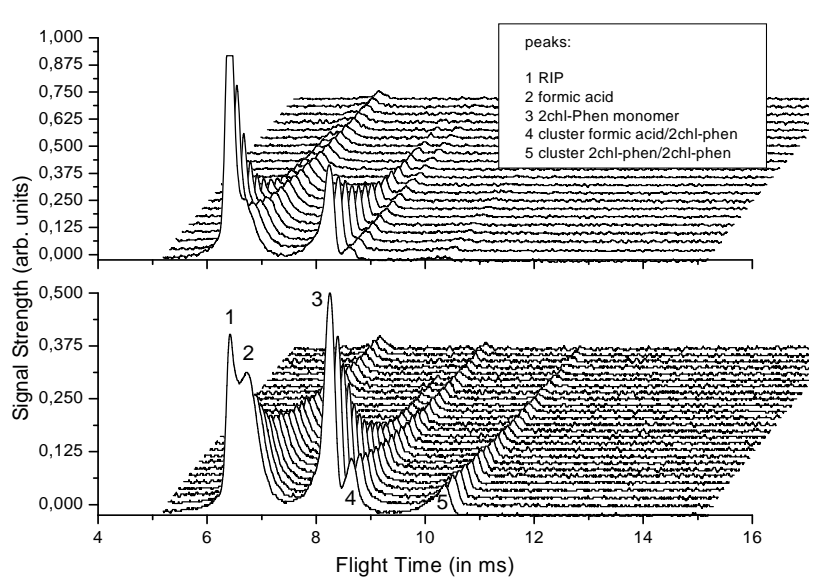

Fig. 4. IMS spectra of a mixture of formic acid $(223 \mathrm{ppbv})$ with 2-chlorophenol (upper graph: 85 ppbv, lower graph: 275 ppbv). An extra peak appears which we interpret as cluster of formic acid and 2-chlorophenol due to its longer decay time (like the cluster of peak 5) compared to the monomer signals (peak 2 and 3). The cluster signal with two 2-chlorophenol moelcules appears only at higher concentrations, similar to the case of a single substance (see fig 2.) The delay time increase in-between two spectra is $100 \mu \mathrm{s}$

\section{CONCLUSIONS}

In this contribution we have analyzed the response of two substances that are typical candidates in pollution monitoring when analyzed with a pulsed ion mobility spectrometer. Besides the single substance spectra we have analyzed the spectra of a mixture of both substances. Similar to the positive mode, additional peaks appear in that case, which seems to be formed by clusters consisting of both analyte molecules, with one present as an ion (in contrast to dimer formation in the positive mode where two analyte neutrals are bound by a proton). Calculations at the B98/6-311++(3df,3dp)// B3LYP/6-31(d) level of theory for the cluster yielded a structure which can be interpreted as a proton bound cluster of analyte anions with a stabilization energy in the typical range for such clusters (which has to be interpreted with care since water clusters have not been included in the calculations). This structure, however, seems to be a special case for formic acid due to its acidic properties. Nevertheless, it could be shown that also in the negative mode using a pulsed electron source can discriminate such peaks from monomer peaks due to their different decay times and thus simplify the interpretation of IMS spectra regarding substance identification. Thus a typical problem of IMS devices when using them in pollution monitoring, the complexity of IMS spectra when analyzing mixtures, can be limited in its effect and thus the performance is increased. Further research needs to analyze different mixtures in order to verify these findings, but the outlook based on the spectra presented here looks already very promising.

\section{REFERENCES}

[1] H. Borsdorf, T. Mayer, M. Zarejousheghani, and G.A. Eiceman, Appl. Spec. Rev, vol. 46, pp. $472-521,2011$.

[2] G. A. Eiceman and Z. Karpas, Ion Mobility Spectrometry, Boca Raton, USA: CRC Press, 2005.

[3] W. Baether, S. Zimmermann, and F. Gunzer, Sens. Act. B, vol. 12, pp. 1238-1243, 2012.

[4] F. Gunzer, A. Ulrich, and W. Baether, Int. J. Ion. Mobil. Spectrom, vol. 13, pp. 9-16, 2010.

[5] F. Gunzer, S. Zimmermann, and W. Baether, Anal. Chem., vol. 82, pp. 3756-3763, 2010.

[6] W. Baether, S. Zimmermann, and F. Gunzer, Int. J. Ion. Mobil. Spectrom, vol. 14, pp. 99-107, 2011.

[7] W. Baether, S. Zimmermann, and F. Gunzer, Rev. Anal. Chem., vol. 31, pp. 139-152, 2012.

[8] L. A. Curtiss, P. C. Redfern, and K. Raghavachari, J. Chem. Phys., vol. 123, pp. 1-12, 2005.

[9] C. J. Smallwood and M. A. McAllister, J. Am. Chem. Soc., vol. 119, pp. 11277-11281, 1997.

[10] J. B. Foresman and A. Frisch, Exploring Chemsitry with Electronic Structure Methods, Pittsburgh, PA, USA: Gaussian Inc., 1996.

Frank Gunzer was educated at University of Kiel, Kiel, Germany Institute of Experimental and Applied Physics during 1993 - 1999. From 1999 to 2003, he completed Dr Degree in Natural Sciences, University of Kiel, Kiel, and Germany Institute of Physical Chemistry Laser Mass Spectrometry Departments. He worked in the Information Engineering and Technology Faculty, German University in Cairo in October 2010 as an associate professor. From 2011 till now, he is Head of Electronics Engineering Department, Information and Technology Faculty, German University in Cairo. 